\title{
Perspektiven der Subjektivität: \\ Das Verhältnis von Systemzeit und Eigenzeit in den perfektischen Tempusformen
}

\author{
Richard Schrodt, Wien
}

1. Die Diskussion zu sprachwissenschaftlichen Themen über Tempus und Zeit ist angesichts der kaum zu bewältigenden Forschungsliteratur ein Wagnis. Die von Klein (1994, S. xii) gegebene Charakteristik der Forschungsliteratur „It is simply impossible to read, let alone to discuss, all possibly relevant literature. This has often been said before (even this has been said before)." wird jede Tempustheorie überdauern. Angesichts dieser Problematik versuche ich, mich auf Grundsätzliches zu beschränken. Ich werde also nicht auf die zahlreichen verschiedenen Formalisierungen eingehen können, und auch bei den verschiedenen Beschreibungsmethoden beschränke ich mich auf das unerlässlich Notwendige. Mir geht es hier um ein Thema, das eher außerhalb der formallinguistischen Forschung steht und daher auch angesichts der intensiven Forschung nicht deutlich wird, nämlich um die Formen und Funktionen der Eigenzeitlichkeit in literarischen Texten. Ich werde mich dazu auf philologisch nachvollziehbare Argumente stützen und tempustheoretische Überlegungen so weit wie möglich auf ein Minimum beschränken.

2. Die Abbildung der zeitlichen Verhältnisse in den Sprachen scheint auf den ersten Blick unproblematisch zu sein: Der Strom der Zeit fließt von der Vergangenheit über die Gegenwart zur Zukunft, demnach müssten nur drei Tempora unterschieden werden. Da das zukünftige Geschehen meist einen modalen Wert hat (es ist erwartet, erwünscht, erhofft oder befürchtet), kann es oft als Modusform angesehen werden. Ein universell gültiges Tempusschema müsste also mit zwei Tempora, Gegenwart und Vergangenheit, auskommen. Tatsächlich kann auch für manche europäischen Sprachen ein Zwei-Tempora-System angenommen werden, unter bestimmten methodischen Voraussetzungen auch für das Deutsche. Tatsache ist aber auch, dass für andere Sprachen eine größere Zahl an Tempuskategorien 
angenommen wird. Es kommt nun darauf an, mit welchen Methoden eine solche Zahl ermittelt wird. Versucht man, Gebrauchskategorien zu erfassen, kommt man zu einer beinahe beliebig großen Zahl von Tempora, übrigens auch für das Deutsche. Führt man hingegen feste Kriterien ein wie etwa die Grammatikalisierung, so lässt sich diese Zahl deutlich reduzieren. Dabei muss natürlich erst bestimmt werden, was unter "Grammatikalisierung“ verstanden werden soll. Im Prinzip erlaubt der Zeitpfeil eine beliebige Unterteilung in kleinere Abschnitte, etwa „unmittelbare Gegenwart“ - „gerade vergangenes Geschehen“ - „unmittelbar bevorstehendes Geschehen“ und innerhalb der Vergangenheit etwa „änger - kürzer vergangenes Geschehen“. Diese Unterteilungen können einzelsprachlich auch durch feste Ausdruckskategorien bezeichnet sein. Eine (beinahe) beliebig feine Unterscheidung kann auch dadurch erreicht werden, dass man sich auf die Folge und das Ineinander von Zeitstufen bezieht (z.B. Harweg 1994). Sprachwissenschaftlich beschreibbare Komplexität entsteht also dadurch, dass auf der konzeptionellen Seite der Sprache verschiedene Schichtungen unterschieden werden können, während auf der Ebene der außersprachlichen Realität oft einfache und klar erkennbare Gegensätze vorhanden sind. Alle diese verschiedenen Beschreibungsmöglichkeiten beziehen sich auf grundlegende Tempuskategorien: Die Unterscheidung zwischen Gegenwärtigem und Vergangenem ist vom Bezeichneten her ebenso vorbestimmt wie etwa die Unterscheidung zwischen der Einzahl und der Mehrzahl (auch hier kann es auf der konzeptionellen Ebene verschiedene Formen der Pluralität geben). Man wird also eine entsprechende Kategorisierung als universalgrammatische Bezeichnungskonstante ansehen können.

3. Ein weithin anerkannter methodischer Ansatzpunkt der Tempuslinguistik ist die Tempuslogik von Reichenbach (1966 [1947]). Derzufolge bezeichnet das Tempus als Basiskategorie des Verbs die zeitliche Beziehung zwischen dem Sprechakt (S) und dem Zeitpunkt oder Zeitintervall des von der Aussage bezeichneten Sachverhalts oder Ereignisses (E). Aus diesen absoluten zeitlichen Relationen ergeben sich die Kategorien Präsens ( $E$ gleichzeitig $S)$, Präteritum ( $E$ vor $S$ ) und Futur $(E$ nach $S)$. Einfachere Tempussysteme wie Nicht-Präteritum können mit $E$ gleichzeitig $S$ oder $E$ nach $S$ bzw. E vor $S$ oder E gleichzeitig $S$ formuliert werden. Das relative Tempus bezieht sich auf einen Referenzpunkt (R), welcher selbst nicht in einem Zeitverhältnis zum deiktischen Zentrum der Rede steht, sondern sich aus dem 
Kontext ergibt. Die Formulierungen entsprechen den absoluten Relationen mit dem Ersatz von $S$ durch $R$. Das Plusquamperfekt ist demnach durch $E$ vor $R$ vor $S$ wiederzugeben (Comrie 1985, 122ff.). Diese auf Reichenbachs Formulierung der universellen Zeitrelationen zurückgehende Beschreibung ist neuerdings in verschiedene Richtungen modifiziert worden (Überblick für das Deutsche bei Thieroff 1992 und d'Alquen 1997; intervallsemantische Fassung bei Klein 1994; mit Welke 2005 liegt nun eine umfassende und umsichtig argumentierende Darstellung vor). Das gilt v.a. für Reichenbachs Referenzpunkt, der ja mit dem Sprechzeitpunkt zusammenfallen kann und damit bei den einfachen Tempora überflüssig wird. Statt dessen sind auch Begriffe wie „Evaluationszeit“ und „Orientierungszeit“ in Gebrauch, die aber alle mehr oder weniger dasselbe meinen, nämlich einen Zeitpunkt (oder ein Zeitintervall), von dem aus das Ereignis betrachtet und zeitlich eingeordnet wird. Man wird auch damit rechnen müssen, dass es mehrere solcher Referenzzeiten in mehreren Schichtungen geben kann. Im Fall der indirekten Rede ist es weiters sinnvoll, einen dem Sprechzeitpunkt vergleichbaren zweiten Zeitpunkt anzunehmen, der nicht mit dem faktischen Äußerungszeitpunkt übereinstimmt, sondern ein zweites deiktisches Zentrum für die eingebettete Aussage bezeichnet. Dafür hat Thieroff (1992) den Terminus „Orientierungszeit“ vorgeschlagen. Da aber der Sprechzeitpunkt im Normalfall eben das erste deiktische Zentrum einer Aussage ist, könnte man auch hier von einer Orientierungszeit sprechen; dann gäbe es eben zwei verschiedene Orientierungszeiten. Ich werde hier die weitere Entwicklung der von Reichenbach ausgehenden Terminologie nicht verfolgen - es besteht sonst die Gefahr, sich im Gestrüpp verschiedener eben nur teilweise übereinstimmender Analysemethoden heillos zu verfangen. Diese Gefahr wird noch dadurch verstärkt, dass unter manchen Termini wie „Betrachtzeit“ ganz Verschiedenes, geradezu Gegensätzliches, verstanden werden kann. In meinem Beitrag werde ich mich einer an Reichenbach orientierten Terminologie bedienen, was mir schon dadurch gerechtfertigt erscheint, dass die Probleme im Reichenbach'schen Originalsystem (z.B. die Formalisierungen der futurischen Tempora) für mein Thema nicht bedeutend sind. Mir geht es hier nur um den Unterschied zwischen Perfekt und Präteritum, und für die Formulierung dieses Unterschied genügt es, für das Perfekt eine eigene Referenzzeit anzunehmen, die im Bereich des Sprechzeitpunkts liegt. Das Präteritum hingegen hat entweder keine Referenzzeit oder, wenn man aus methodischen Gründen von einer universalgrammatischen Referenzzeit ausgeht, diese Referenzzeit liegt im Bereich der Ereigniszeit. 
4. Mit dieser Formalisierung wird eine Funktion des Perfekts wiedergegeben, die traditionell etwa so formuliert wird, dass das perfektische Geschehen eine Auswirkung oder Nachwirkung in der (Sprecher-)Gegenwart habe. Es handelt sich um den typischen Fall des Resultatsperfekts: Ein Satz wie Ich habe das Buch gelesen soll bedeuten, dass das Buch als Gelesenes gesehen wird und dass mir sein Inhalt in irgendeiner Form präsent ist, während ein Satz wie Ich las das Buch auf das bloß vergangene Geschehen verweist und somit für die Nachwirkung dieser Handlung nichts aussagt. Solche Formulierungen sind aber vage und beziehen sich auf psychische Einstellungen und Vorgänge, die formalgrammatisch schwer nachvollziehbar sind. Die Problematik solcher Formulierungen zeigt sich besonders deutlich in der Geschichte der grammatischen Beschreibungen. Die derzeit neueste Auflage der Duden-Grammatik $(2005,514)$ drückt sich hier sehr vorsichtig aus: „Wenn nichts dagegen spricht, darf denn auch davon ausgegangen werden, dass das Geschehen aufgrund seiner Folgen oder der an ihm beteiligten Aktanten im Sprechzeitpunkt (noch) von Belang ist." An einer anderen Stelle (519) ist davon die Rede, dass „der Kontext das Geschehen oder dessen Folgen als relevant im Sprechzeitpunkt ausweist.“ Diese Formulierungen zeigen sehr gut die Problematik dieser Tempuskategorie im Deutschen. Folgende Konstellationen sollen für den Perfektgebrauch relevant sein:

1. die Eigenschaften des Geschehens selbst,

2. die Rolle der beteiligten Akteure,

3. der „Kontext“, also die sprachliche (und vermutlich auch außersprachliche) Umgebung des betreffenden Satzes, und schließlich

4. eine nicht näher bestimmte „Relevanz“, von der man nur vermuten kann, dass sie ein Merkmal der Aussageabsicht und damit der Sprecherintention ist.

Es werden also nebeneinander Merkmale aus dem Bereich des Signifikats, der Textumgebung und der Sprecherhaltung berücksichtigt, wobei im Bereich des Signifikats nochmals zwischen Geschehensmerkmalen an sich und den beteiligten Personen (Akteuren) unterschieden wird. Besonders vage ist der Bezug auf den Kontext, denn hier kann ein ganzes Bündel von unterschiedlichen Merkmalen bis hin zu verschiedenen Textsorten gemeint sein. Die Problematik der Funktionsbeschreibung des deutschen Perfekts zeigt sich auch deutlich in der ersten Auflage der Duden-Grammatik (1959, 109), die methodisch der inhaltbezogenen Sprachwissenschaft nahe steht: 
Diese Formen bezeichnen meist Vergangenes, das sich noch irgendwie auf die Gegenwart des Sprechenden bezieht, ein Geschehen, das sich erst in der Gegenwart vollendet hat, Geschehnisse der jüngsten Vergangenheit. Der Sprecher wendet den Blick von der Gegenwart aus in die Vergangenheit zurück:

„Es hat geschneit!“ ruft ein Junge, der früh am Fenster den in der Nacht gefallenen Schnee erblickt. Ebenso: Ich bin eben aus der Stadt gekommen. Jetzt babe ich den Schlüssel gefunden. Inge ist heute geprüft worden. Gestern ist ein Kind ertrunken.

Das gleiche Verhältnis ist auch dann gegeben, wenn sich der Sprecher so in die Vergangenheit zurückversetzt, daß ihm ein historisches Ereignis als gerade vollendet erscheint [...]. Dies ist fast immer der Fall, wenn ein vergangenes Ereignis für sich betrachtet werden soll [...]:

Kolumbus hat Amerika entdeckt. Die Burg ist im 15. Jahrhundert erbaut worden. Dies gilt auch, wenn der Sprecher Allgemeingültiges im Perfekt ausdrückt [...]: Ein Unglück ist bald geschehen.

Gegenüber der Neufassung treten zwei Momente stärker hervor: 1. die zeitliche Nähe zur Gegenwart und 2. eine besondere Sprecherhaltung, ein Rückblick oder eine Rückversetzung in die Vergangenheit, also Merkmale, die in der Neufassung zu den Punkten 1 und 4 gehören, wobei besonders das zweite Merkmal das recht vage Kriterium der „Relevanz“ mit etwas konkreteren Inhalten erfüllt. Immerhin wagt die Duden-Grammatik von 1959 eine genauere Beschreibung der psychischen Bedingungen für die Tempussetzung, ein deutlicher Vorteil der inhaltbezogenen Ansätze. Nimmt man alle diese Merkmale zusammen, so zeigt sich aber, dass die Merkmale auf der Ebene des temporalen Signifikats so undeutlich formuliert sind, dass sie wenig zur Erhellung des perfektischen Referenzbereiches beitragen. Etwas besser steht es mit den Merkmalen, die sich auf die Sprecherintention beziehen. Aber auch hier ist die alte Duden-Grammatik genauer, denn mit „Rückblick“ und „Rückversetzung“ sind zwei Sprecherhaltungen genannt, die im Grund psychologisch nachvollziehbare und damit klarer beschreibbare Kategorien sind. Dafür drängt sich geradezu der Terminus „Aspekt“ auf, doch davon soll noch später die Rede sein.

5. Wenn man beim Überbegriff „Relevanz“ bleibt, so ist damit gemeint, dass das Perfekt eigentlich eine modale Kategorie ist, gewissermaßen ein 
verstärkter Indikativ, mit dem die Aktualität des Geschehens deutlich hervorgehoben wird. Kann das deutsche Perfekt tatsächlich eine Moduskategorie sein? So sieht es jedenfalls Engel (1992). Zunächst zur morphologischen Ausgrenzung. Herkömmlich werden 4-6 Tempora unterschieden. Tempora sind Verbformen und haben mit der Zeit zu tun. Es gibt aber unterschiedliche Ausdrucksformen und Bedeutungsschwankungen. Geht man von sechs Tempora aus, so erscheinen sie in zwei Verbformen (Präsens, Präteritum) und vier verbalen Komplexen, davon drei zweigliedrig (Perfekt, Plusquamperfekt, Futur) und einer dreigliedrig (Futur II). Das sind aber natürlich verschiedenartige Konstruktionen. In den klassischen Sprachen (Latein, Altgriechisch) sind alle Tempora (reine) Verbformen. Es liegt daher nahe, hier eine Übernahme der Struktur der klassischen Sprachen auf das Deutsche zu vermuten. Zur semantischen Ausgrenzung: Herkömmlich wird angenommen, dass Informationen über Zeitverhältnisse bezeichnet werden. Aber bekanntlich ist das Präsens zeitindifferent, so wird es jedenfalls in vielen Grammatiken dargestellt. Das Futur hat oft (meist) „modale" Bedeutung (Er wird krank sein), das Futur II ebenso (Sie wird es getan baben). Daher ist nach Engel der Zeitwert fraglich. Zeitliche Informationen werden auch durch andere Konstruktionen bezeichnet (ist am Bauen, will bauen, soll bauen, hofft zu bauen usw.). Daher liegt die Schlussfolgerung nahe: Zeitlichkeit ist mehr als die Tempora, und einige Tempora sind nicht oder nur eingeschränkt zeitlich zu verstehen. Der weitere Schluss: Tempora lassen sich nicht semantisch definieren. Schließlich kann man fragen, ob es überhaupt verbale Ausdrücke gibt, die sich semantisch ausgrenzen lassen. Nach Engel sind es die finiten Verben. Sie sagen etwas über die Realität des entsprechenden Sachverhalts aus, im Gegensatz zu den infiniten Verbformen. Dazu kommen noch zeitliche Bedeutungen bei bestimmten finiten Verbformen: Präteritum - Vergangenheit, Imperativ - Zukunft. Im Präsens wird ein Sachverhalt einem bestimmten Zeitraum oder Zeitpunkt zugeordnet, doch bleibt dieser Raum/Punkt grundsätzlich offen. Eine weitere semantische Kategorie zeigt sich am folgenden Beispiel: Alexander zerschnitt/zerschneidet den gordischen Knoten. Sind diese Sätze bedeutungsgleich? Tatsächlich bezeichnen beide Sätze den Sachverhalt als real. Normalerweise wird der erste Satz als ein Beispiel für das „historische Präsens“ angeführt: Ein vergangener Sachverhalt wird in die Gegenwart hereingeholt, wird gegenwärtig gemacht. Das ist aber nur möglich, wenn man das Präsens als „Gegenwart" interpretiert; doch das wird bekanntlich bestritten. Hingegen kann man beim Satz im Präsens behaupten, dass der beschrie- 
bene Sachverhalt dem Sprecher näher steht als beim Satz im Präteritum. Das Präsens signalisiert also, dass ein Sachverhalt die Gesprächsbeteiligten unmittelbar angeht, für sie von Belang ist, und gehört damit einer modalen Dimension an. Dazu kommt noch das Merkmal des Gesprächspartnerbezugs, das ich aus Engels großer Grammatik (Engel 2004) ergänzt habe. In einer Tabelle zusammengefasst:

\begin{tabular}{|l|c|c|c|c|}
\hline Verbform & Realität & Zeit & $\begin{array}{c}\text { Modali- } \\
\text { tät }\end{array}$ & $\begin{array}{c}\text { Gesprächspartner- } \\
\text { bezug }\end{array}$ \\
\hline Präsens & real & bestimmt, aber offen & + & belangreich \\
\hline Präteritum & real & vergangen & 0 & nicht belangreich \\
\hline Perfekt & $\begin{array}{c}\text { real } \\
\text { abgeschlos- } \\
\text { sen }\end{array}$ & $\begin{array}{c}\text { bestimmt, aber zeitlich } \\
\text { nicht festgelegt }\end{array}$ & + & belangreich \\
\hline
\end{tabular}

6. An dieser auf den ersten Blick durchaus ansprechenden neuen Sicht des deutschen Verbalsystems fällt zunächst auf, dass Engel im Gegensatz zur grammatischen Tradition eine andere Auffassung von Modalität hat: Der Sachverhalt ist für die Gesprächspartner belangvoll. Die traditionelle Auffassung hingegen beruht letzten Endes auf dem Zusammenhang mit dem Status der Aussage als logisches Urteil (ein Erbe der logizistischen Grammatik). Diese Auffassung erlaubt eine klare inhaltliche Charakterisierung: Wenn ein Urteil ausgesprochen wird, dann steht der Indikativ; wenn kein Urteil (sondern Wunsch, Wille, Befehl, Möglichkeit, Unsicherheit usw.) ausgesprochen wird, dann kann der entsprechende Satz ein grammatisches Zeichen für die Modalität enthalten (Konjunktiv, Imperativ) oder diese Modalität ist lexikalisch bezeichnet (Modalpartikeln, Satzadverbien). D.h. in der traditionellen Sicht ist der verbale Modus eine grammatische Form, welche die durch den Sprecher angezeigte Geltung der Aussage bezeichnet. Für diesen Inhalt führt Engel aber eine eigene Kategorie der Realität ein, die vom Status der Verbmorphologie abhängt (finites/infinites Verb). Die traditionelle Bindung des Modus an die Urteilsqualität eines Satzes wird damit aufgegeben. Die verbalen Kategorien werden enger an die Verbgestalt gebunden; die satzzentrierte Fassung der semantischen Verbkategorien wird durch die verbzentrierte Fassung dieser Kategorien ersetzt. 
7. Das gibt an sich einen guten Sinn: Eine Grammatik als endliches Inventar von sprachlichen Zeichen verlangt auch eine Formgebundenheit der Beschreibung. Insofern ist es durchaus verständlich, wenn die zusammengesetzten Verbformen nicht zum Kernbereich der Grammatik gestellt werden. Weiters wird das Verb als strukturelles Zentrum des Satzes konsequent hervorgehoben, und das ist zweifellos eine sinnvolle Methode der grammatischen Beschreibung des Deutschen. Der spezifische Ansatz dieser Kategorisierung zeigt sich besonders deutlich am viel diskutierten Verhältnis von Perfekt und Präteritum. Nach Engel gibt es keine Übergänge zwischen diesen beiden „Tempora“ (auch im Original in Anführungszeichen) und daher auch weder Verwechslungsmöglichkeiten noch Zweifel über ihre Anwendung: „[E]in präteritaler Vorgang ist vergangen und ohne Belang für die Gesprächsbeteiligten, ein perfektischer Vorgang hingegen ist abgeschlossen und für die Gesprächsbeteiligten belangvoll.“ (Engel 1992, 62) Wenn auch kompetente Deutschsprecher „sich fragen, ob ,abgeschlossen“ und ,vergangen" nicht zwei Namen für dieselbe Sache seien“, dann handelt es sich möglicherweise um eine Neutralisierung dieser Tempusopposition in bestimmten Nebensätzen wie Weißt du noch, wann sie bier eintraf/eingetroffen ist? Zum berühmten Schluss aus Goethes „Werther" Handwerker trugen ibn. Kein Geistlicher hat ibn begleitet. erklärt Engel: „Demnach vermittelt der erste Satz, aus der Distanz gesehen, eine Hintergrundinformation. Der zweite aber bringt das Wesentliche, das den Leser anspringt, ihm keine Gelegenheit läßt, sich aus der Situation zu stehlen, sich unbeteiligt zurückzulehnen. Dies geht ihn unmittelbar an. Hier ist nichts austauschbar. [...] Und im Grunde sind, sorgsam verwendet, Perfekt und Präteritum überhaupt nie austauschbar." (Engel 1992, 63) Man mag von Engels Tempustheorie halten, was man will: Aber nur sie erlaubt es, futurische Gebrauchsarten des Perfekts wie In einer halben Stunde habe ich den Brief geschrieben in ein argumentierbares Beschreibungssystem einzugliedern. Andernfalls müsste man die Orientierungszeit für diese Gebrauchsart nach die Sprecherzeit verschieben, was zwar formal durch die Koppelung der Orientierungszeit mit temporaldeiktischen Ausdrücken möglich ist, aber die Generalisierbarkeit der Tempusbeschreibung deutlich einschränkt.

8. Engels Tempustheorie hat sich nicht durchgesetzt. Sie ist aber für unser Thema von entscheidender Bedeutung, zeigt sie doch sehr deutlich die Ursachen für die Problematik der wissenschaftlichen Beschreibung des 
deutschen Tempus. In allen Modellen, die sich an Reichenbach orientieren und die ich hier mit der Bezeichnung „deiktisch“ benenne, ist das deutsche Perfekt (und universalgrammatisch jedes Perfekt) ein Vergangenheitstempus, denn das bezeichnete Ereignis liegt nun einmal vor der Referenzzeit bzw. vor der Orientierungszeit. Im „Abweichungsmodell“ von Engel (und in ähnlichen, nicht-deiktischen Modellen wie etwa bei Weinrich) ist das deutsche Perfekt ein „Gegenwarts“tempus, wobei über ein universalgrammatisches Fundament nichts gesagt wird. Es scheint so, als würden sich theoretisch die beiden grammatischen Komponenten des Perfekts, das Präsens des Hilfsverbs und das Präteritum des Partizips, verselbstständigen lassen. An diesem Punkt ist es nun unvermeidlich, den Terminus „Aspekt“ ins Spiel zu bringen. Aspektpaare, welcher Art auch immer, werden normalerweise mit den Termini „perfektiv - imperfektiv“ belegt. Ich gebrauche hier eine andere Terminologie: „Durativ“ ist der Aspekt, unter dem eine Handlung in ihrer Dauer gesehen wird; Anfang und Ende der Verbalhandlung kommen nicht in das Blickfeld. Demgegenüber ist „komplexiv“ der Aspekt, unter dem eine Verbalhandlung als unteilbare Ganzheit gesehen wird. Im Neuhochdeutschen (Nhd.) ist der Aspektunterschied nicht grammatikalisiert; er kann durch Fügungen wie etwa einen Pullover stricken (kursiv) und einen Pullover fertig stricken (komplexiv) verdeutlicht werden (Wortpaare wie etwas das bekannte jagen - erjagen geben hingegen einen Aktionsartunterschied wieder).

9. Aspektunterschiede können durch eine Vielzahl von grammatischen Formen kodiert sein. Im Nhd. stehen auch Formunterschiede wie an einem Brief schreiben / einen Brief schreiben und den Brief schreiben zur Verfügung: Im ersten Fall ist eine andauernde Handlung bezeichnet, die zwar Anfang und Ende hat, aber ohne dass diese Begrenzung kommunikativ wichtig wäre. Deshalb kann sich diese Phrase mit durativen Zeitadverbien wie stundenlang verbinden. Im letzten Fall ist das Ergebnis der Handlung, der geschriebene Brief, wichtig. Zeitadverbien wie heute beziehen sich auf die Gesamtheit der Verbalhandlung. In sie schreibt stundenlang den Brief (besser: sie schreibt den Brief stundenlang) überlagert das durative Zeitadverb den komplexiven Verbalhandlungskern mit dem Ergebnis einer kursiven Verbalphrase (daher steht auch das Zeitadverb in Ausdrucksstellung am rechten Satzrand). Objektiv liegt derselbe Sachverhalt vor, nur die Sichtweise ist eine Andere. Insofern kann man tatsächlich davon ausgehen, dass der As- 
pekt eine subjektive grammatische Kategorie ist. Es gibt aber grammatische Erscheinungen, die bestimmte Aspektformen begünstigen, sodass auf diese Weise eine Grammatikalisierung angebahnt sein kann. In nhd. Kausalsätzen z.B. ist in der Standardsprache das (aspektuelle) Perfekt üblicher als das Präteritum, weil die Verbalhandlung nicht in ihrem Verlauf und in ihrer Ausdehnung, sondern nur als punktuell gesehenes kausales Moment wichtig ist. Ein Kausalsatz im Präteritum ist aber keineswegs falsch: Gelegentlich ist gerade der Handlungsverlauf das kausal wichtige Moment, etwa in Sie war damals sicher zu Haus, weil in ibrer Wohnung die ganze Zeit das Licht brannte. Es können also auch in der Sprache Aspekte, besser gesagt: aspektuelle Formen, vorhanden sein, wenn dafür kein geschlossenes und systematisches Bezeichnungsinventar besteht. Die wissenschaftliche Kontroverse lässt sich damit auf einen einfachen gemeinsamen Nenner bringen: In deiktischen Tempussystemen ist das Perfekt ein Vergangenheitstempus; man braucht den Aspekt als Beschreibungskategorie nicht. Demzufolge hat das (deutsche) Perfekt keine aspektuelle Funktion. Das ist die gängige Meinung. In nicht-deiktischen Tempussystemen ist das Perfekt eine aspektuelle Kategorie (selbst wenn das manche Forscher wie Weinrich nicht wahrhaben wollen); man braucht das Tempus als Beschreibungskategorie nicht. Natürlich können Aspekt und Tempus kombiniert werden, weil es sich um prinzipiell voneinander unabhängige Kategorien handelt. Das deutsche Perfekt mag ein Tempus sein: Sobald man aber Ausdrücke wie subjektive Perspektive, Sicht von Außen, Totalität, Definitheit, Rückschau/Vorschau usw. gebraucht, kommt zum Tempus eine aspektuelle Qualität hinzu. Das Perfekt ist somit eine Art „subjektive Zeit“, oder genauer: Es kann zur Bezeichnung einer „subjektiven Zeit“ verwendet werden. Diese Ansicht wird auch in der neueren Forschung vertreten (z.B. Marschall und Valentin in Quintin/Najar/Genz 1997); diese Ansicht wird auch neuerdings von Welke $(2005,250)$ bestätigt: „Es geht um subjektive Eindrücke, um Interpretationen und Auslegungen [...]“. Die Subjektivität des Perfekts, die ja schließlich auch ein textuelles Merkmal sein kann (dazu Confais 1995, Kap. 3), führt zu meinem eigentlichen Thema, der Eigenzeitlichkeit in der Sprache.

10. Ich führe den Begriff der Eigenzeitlichkeit mit einem Zitat aus dem Buch von Jakob von Uexküll und Georg Kriszat, Streifzüge durch die Umwelten von Tieren und Menschen, 1956, ein (30): „Die Zeit, die alles Geschehen umrahmt, scheint uns das allein objektiv Feststehende zu sein 
gegenüber dem bunten Wechsel ihres Inhaltes, und nun sehen wir, dass das Subjekt die Zeit seiner Umwelt beherrscht. Während wir bisher sagten: Ohne Zeit kann es kein lebendes Subjekt geben, werden wir jetzt sagen müssen: Ohne ein lebendes Subjekt kann es keine Zeit geben."Uexküll erläutert diese Eigenzeitlichkeit am Beispiel der Wahrnehmungsreize: Ein Moment als kleinster Zeitraum, innerhalb dessen alle Reize unabhängig von ihrer objektiven Zeitfolge als gleichzeitig empfunden werden, dauert beim Menschen eine 18tel-Sekunde, bei der Weinbergschnecke eine Viertelsekunde, bei der Pilgermuschel 13 Sekunden und bei der Zecke, die bis zu 18 Jahre ohne Nahrung in einem schlafähnlichen Zustand auf ihr Opfer warten kann, noch viel länger. Diese subjektive Zeit hängt bei den Lebewesen vom Rhythmus des Zentralnervensystems ab. Innerhalb der Impulse des Nervensystems steht die Zeit für das Lebewesen gewissermaßen still. Es ist klar, dass eine solche Vorstellung nur metaphorisch auf die sprachliche Gestaltung von Geschehnissen übernommen werden kann. Es gibt aber, so behaupte ich, grundsätzliche Übereinstimmungen von biologisch beschreibbaren Wahrnehmungsakten und sprachlichen Gestaltungsmöglichkeiten, die es erlauben, recht genau an den Begriff der Eigenzeitlichkeit bei Uexküll anzuknüpfen - abgesehen von den verschiedenen weiteren Verwendungsweisen dieses Begriffes, z.B. im Bereich der Sozialwissenschaften (vgl. etwa Nowotny 1993) oder in der Chemie und der Physik. Auch in der sprachlichen Gestaltung gibt es also so etwas wie einen erzählpraktischen Stillstand der Zeit, einen Zeitpunkt, an dem das erzählte Geschehen zu einer Art „Nullpunkt“ gerinnt und aus diesem Nullpunkt heraus eine neue Gestalt und damit auch eine neue Wirkungsweise gewinnt. Man muss nur Uexkülls „lebendes Subjekt“ durch das „sprechende/erzählende Subjekt“ ersetzen; allerdings stehen diesem „redenden Subjekt“, wie ich es hinfort zusammenfassend nennen will, grundsätzlich immer zwei Wahrnehmungsweisen = Erzählweisen zur Verfügung: die kontinuierliche, von Zeitpunkt zu Zeitpunkt (von Ereignis zu Ereignis) fortschreitende Erzählung und die momenthafte, das Geschehnis auf einen Punkt bringende „Totalfassung“. Diese „Totalfassung“ entspricht damit geradezu einem erzählpraktischen Wahrnehmungsakt: Hier sind nicht die einzelnen Ereignisse und ihre Folge wichtig, sondern das, was von diesen einzelnen Ereignissen als Eindruck und Wirkungsmoment auf das sprechende/erzählende Subjekt bleibt und was er/sie dem/der Leser/in als besonders hervorgehobene Aussage mitteilen will. Man kann das ein „resümierendes“ Perfekt nennen (vgl. dazu Vater 1997). Dem Rhythmus des Zentralnervensystems entspricht im Erzählakt 
der Rhythmus der Aussagegewichtung: Einzelne Begebenheiten verdichten sich zu einem Moment, das in seiner Bedeutung über diese Begebenheiten hinausreicht. Charakteristisch für die Sprache ist freilich, dass dieser Rhythmus rekursiv ist, d.h. er stellt sich auf verschiedenen Stufen der Komplexität in der gleichen Weise, aber in verschiedenen konkreten Formen, ein. Eines dieser Zeichen für den erzählpraktischen Nullpunkt ist das Perfekt.

11. Man kann diese Erzählpraxis nur an ganzen Texten sinnvoll erläutern, und das macht eine Demonstration schwierig. Ich werde mich daher hier auf zwei kurze Texte beschränken, auf Kürzestgeschichten, wie man sie auch genannt hat. Der erste Text stammt von Walter Jens und hat den Titel „Bericht über Hattington“. In dieser Geschichte in Form eines Erlebnisberichts wird erzählt, wie der Ausbruch eines Verbrechers mit dem Namen Hattington das Leben in einer Kleinstadt verändert. Das Grundtempus ist das Präteritum. Die Geschichte beginnt mit dem folgenden Absatz:

Der Winter kam in diesem Jahr sehr früh; schon Mitte November hatten wir 15 Grad Kälte, und in der ersten Dezemberwoche schneite es sechs Tage lang hintereinander; am fünften, einem Mittwoch, brach Hattington aus. Er hatte offenbar damit gerechnet, daß der Schnee seine Spuren verschluckte - und diese Rechnung ging auf. Die Hunde verloren die Witterung, und die Gendarmen kehrten noch im Laufe der Nacht nach Colville zurück. Am Morgen darauf wurde unser Polizeiposten verstärkt, und Sergeant Smith bekam zwei neue Kollegen: man vermutete nämlich, daß Hattington versuchen würde, auf dem schnellsten Wege zu uns nach Knox zu gelangen; denn hier hatte man ihn, einen seit langem gesuchten Verbrecher, im Mai auf offener Straße verhaftet - wahrscheinlich auf eine Anzeige hin, die von der Kellnerin Hope und dem Tankstellenwart Madison kam, bei denen Hattington in Kreide stand. Die Annahme lag also nahe, daß der Zuchthäusler, um Rache zu nehmen, zuerst nach Knox kommen würde.

Das Plusquamperfekt ist hier und in den folgenden Stellen der Ausdruck der Vorzeitigkeit, also ein deiktisches Tempus, das ein Ereignis bezeichnet, welches vor dem Geschehen der Haupthandlung liegt. Im nächsten Absatz werden die vergebliche Suche nach dem Verbrecher und das aufkommende allgemeine Misstrauen beschrieben, jemand hätte Hattington 
versteckt. Doch als bis über Weihnachten nichts geschah, kam das Leben in der Kleinstadt wieder in seine gewohnten Bahnen. Im folgenden Absatz wird berichtet, wie ein Mord und eine Misshandlung eines jungen Mädchens, für die Hattington verantwortlich gemacht wurde, das Leben in der Stadt aus dem Gleis warfen. Die Verdächtigungen kamen verstärkt wieder auf, es kam zu einer Hexenjagd und zu Randalen:

Bald gab es kein Geheimnis mehr, das, von Schnüfflern entdeckt, nicht ans Tageslicht kam: Ehemänner, die einmal gefehlt hatten, sahen sich wie Verbrecher behandelt, harmlose Trinker wurden des Mordes verdächtigt; der Frauenverein ließ vor den Kino-Vorstellungen Zettel verteilen, auf denen sich die Bürger ermahnt sahen, den Umgang mit gewissen Leuten, wenn ihnen das Leben lieb sei, zu meiden. Auf der anderen Seite mehrten sich gerade in diesen Tagen unter den jungen Leuten Unordnung und Zuchtlosigkeit. Während die Älteren ihre Häuser nach Möglichkeit nur noch zur Arbeit oder zum Kirchgang verließen, versammelten sich die Jüngeren abends im Wirtshaus, tranken und johlten, pöbelten die Erwachsenen an und errichteten am Ende ein solches Schreckensregiment, daß wir ihrer nur mit Hilfe einer Art von Zivilpolizei, der Bürgerwehr, Herr werden konnten. Schließlich blieb kein anderer Ausweg, als die Rädelsführer kurzweg zu verhaften - und dabei kam dann heraus, daß auch die schlimmsten Radaubrüder sich eher aus Furcht, eines Tages Hattingtons Opfer zu werden, denn aus Übermut zusammenrotteten.

Dieser Stelle folgt nun ein Satz im Perfekt: Das hat mir wieder einmal gezeigt, wie schnell die allgemeine Raserei im Schatten der Angst und des Schreckens gedeibt. Dieser Satz könnte auch im Präteritum stehen und wäre damit nicht auffällig. Das Perfekt bezeichnet hier aber nicht ein Geschehen, sondern den Eindruck des Geschehens auf den Sprecher/Erzähler. Dieser Eindruck wird noch durch das Prädikatsverb zeigen und die Ausdrücke der subjektiven Modalität mir wieder einmal bestätigt. Auch der folgende Objektsatz, der eine allgemein-menschliche und damit nicht auf die Ereigniszeit bezogene Verhaltensweise weist, verstärkt diesen Funktionsbereich des Perfekts. Man hat den Eindruck, diese Geschichte wurde eben deshalb erzählt, um genau diese Aussage zu belegen. Damit könnte sie auch schon zu Ende sein.

Aber es wird (im Präteritum) weiter erzählt: Auch die Eltern standen im Radaumachen ihren Kindern nicht nach. Es folgt wieder ein Satz im Perfekt: Ich selbst habe Nächte erlebt, in denen man mich mebr als ein dutzend- 
mal anrief, um mich mit verstellter Stimme zum Boykott angeblich verdächtiger Bürger zu zwingen. Wieder wäre eine präteritale Fassung (Ich selbst erlebte Nächte, in denen ...) nicht auffällig. Doch auch hier weist das Subjektspronomen in der 1. Person auf ein Begebnis, das sich aus der Folge der erzählten Ereignisse durch seine Sprecherbezogenheit heraushebt; nur handelt es sich hier um ein Begebnis, das in die Folge der berichteten Ereignisse eingebettet ist. Dieses Begebnis entzieht sich aber einer strikten Folgebeziehung: Es sind eben mehrere Nächte gemeint, die über die gesamte Ereigniszeit dieses Absatzes verstreut sind. Hier kann die Geschichte nicht zu Ende sein, sie geht weiter: Im darauf folgenden Absatz wird berichtet, dass am 17. März ein zweiter Mord geschah. Es kam zu hysterischen Ausschreitungen gegenüber grundlos Verdächtigten. Der nächste Absatz beginnt wieder mit einem Satz im Perfekt, das im nächsten Satz sofort ins Präteritum wechselt: Im April hat dann sogar Reverend Snyder, einer der letzten besonnenen Männer, kapituliert: von der Kanzel aus befabl er uns, den Mörder und seine Helfershelfer zu jagen. Von den perfektischen Sätzen könnte dieser Satz wohl am unauffälligsten ins Präteritum umgeformt werden. Hier zeigt sich tatsächlich das, was die neue Duden-Grammatik unter Merkmale des Geschehens und der beteiligten Akteure versteht: Das Geschehen erreicht seinen Höhepunkt, die Verfolgungsjagd wird durch einen bisher besonnenen Akteur abgesegnet. Ich verstehe dieses Perfekt daher nicht als Ausdruck einer Eigenzeit, sondern als textuell gebundenes Zeichen.

Doch dann wird eine unerwartete Wende berichtet. Die Schneeschmelze begann.

Die Sonne brachte alles an den Tag: am Karfreitag fand man Hattingtons Leiche, hundert Meter vom Zuchthaus entfernt. Weiter war er nicht gekommen bei seinem Ausbruchsversuch im Dezember. Der Schnee hatte die Spuren verschluckt, der Eissarg seinen Körper geschützt.

Der letzte Absatz lautet:

Von diesem Tage an begann es still zu werden, hier bei uns in Knox. Wer es irgend ermöglichen konnte, zog weg. Emily Sawdys und Madisons Mörder aber wurde niemals gefunden, das Vergehen an Helen Fletcher nicht gesühnt. Nur ich habe einen bestimmten Verdacht, doch ich schweige, und sonst weiß niemand, wer der Täter war. Eines aber ist sicher: es gibt nicht viele Leute in unserer Stadt, die frei sind von Schuld. 
Die Erzählung endet mit drei Sätzen im Präsens. Die ersten zwei beziehen sich auf Verhaltensweisen des Erzählers, der letzte bezeichnet eine Tatsache, die sich aus der Geschichte ergibt, und formuliert damit die poetische Botschaft dieses Textes. Die Geschichte kehrt so zum Erzähler zurück.

12. Das Perfekt als ein Moment der Subjektivität ist besonders charakteristisch für Erzählabschnitte, in denen ein Resümee gegeben oder eine allgemeine Folgerung gezogen wird. Solche Erzählabschnitte können Texte gliedern und sie zu einer Folge von verschiedenen Sinneinheiten strukturieren. Perfektformen kommen oft in einer Koda vor: Sie bezeichnet das Ende der Geschichte ausdrücklich oder enthält allgemeine Folgerungen und Beobachtungen, die sich aus der Geschichte ergeben (die „Moral“). Das, was in der Koda steht, tritt aus dem zeitlichen Rahmen der übrigen Erzählteile heraus und ist stärker mit dem Hier und Jetzt des Erzählenden verbunden. Derartige Textabschnitte können auch in manchen Textsorten am Anfang stehen, so z.B. in Agenturmeldungen von Tageszeitungen (Marschall 1995, Schecker/Padros/Jechle 1997). Ein besonders interessantes Beispiel für perfektische Ausdrücke am Anfang eines Textes ist die Erzählung „Seine k. und k. apostolische Majestät" von Joseph Roth:

Es war einmal ein Kaiser. Ein großer Teil meiner Kindheit und meiner Jugend vollzog sich in dem oft unbarmherzigen Glanz seiner Majestät, von der ich heute zu erzählen das Recht habe, weil ich mich damals gegen sie so heftig empörte. Von uns beiden, dem Kaiser und mir, babe ich recht behalten - was noch nicht heißen soll, daß ich recht hatte. Er liegt begraben in der Kapuzinergruft und unter den Ruinen seiner Krone und ich irre lebendig unter ihnen herum. Vor der Majestät seines Todes und seiner Tragik - nicht vor seiner eigenen - schweigt meine politische Überzeugung und nur die Erinnerung ist wach. Kein äußerer Anlaß hat sie gerweckt. Vielleicht nur einer jener verborgenen, inneren und privaten, die manchmal einen Schriftsteller reden heißen, ohne daß er sich darum kümmerte, ob ihm jemand zuhört.

Dieser Einleitungsteil gehört zum Hier und Jetzt des Erzählers. Der Autor hat heute das Recht vom dem zu erzählen, was ihm damals empörte. Das, was damals geschah, und das, was heute ist, erscheint auf schicksalshafte Weise verwoben, der Tod des Kaisers und das lebendige Herumirren des 
Erzählers, ein halt- und zielloses Herumirren, so als hätte der Tod der Majestät auch dem Erzähler den Boden entzogen. Es bleibt die Erinnerung. Sie drängt sich auf, sie braucht keinen Anlass, sie verschafft sich die Sprache des Dichters. Das, was folgt, ist die Rechtfertigung für das, was geblieben ist. Ich zitiere die nächsten beiden Absätze:

Als er begraben wurde, stand ich, einer seiner vielen Soldaten der Wiener Garnison, in der neuen feldgrauen Uniform, in der wir ein paar Wochen später ins Feld gehen sollten, ein Glied in der langen Kette, welche die Straßen säumte. Der Erschütterung, die aus der Erkenntnis kam, daß ein historischer Tag eben verging, begegnete die zwiespältige Trauer über den Untergang eines Vaterlandes, das selbst zur Opposition seine Söhne erzogen hatte. Und während ich es noch verurteilte, begann ich schon, es zu beklagen. Und während ich die Nähe des Todes, dem mich noch der tote Kaiser entgegenschickte, erbittert maß, ergriff mich die Zeremonie, mit der die Majestät (und das war: Österreich-Ungarn) zu Grabe getragen wurde. Die Sinnlosigkeit seiner letzten Jahre erkannte ich klar, aber nicht zu leugnen war, daß eben diese Sinnlosigkeit ein Stück meiner Kindheit bedeutete. Die kalte Sonne der Habsburger erlosch, aber es war eine Sonne gewesen.

An dem Abend, an dem wir in Doppelreihen in die Kaserne zurückmarschierten, in den Hauptstraßen noch Parademarsch, dachte ich an die Tage, an denen mich eine kindische Pietät in die körperliche Nähe des Kaisers geführt hatte, und ich beklagte zwar nicht den Verlust jener Pietät, aber den jener Tage. Und weil der Tod des Kaisers meiner Kindheit genauso wie dem Vaterland ein Ende gemacht hatte, betrauerte ich den Kaiser und das Vaterland wie meine Kindheit. Seit jenem Abend denke ich oft an die Sommermorgen, an denen ich um sechs Uhr früh nach Schönbrunn hinausfuhr, um den Kaiser nach Ischl abreisen zu sehen. Der Krieg, die Revolution und meine Gesinnung, die ihr recht gab, konnten die sommerlichen Morgen nicht entstellen und nicht vergessen machen. Ich glaube, daß ich jenen Morgen einen stark empfindlichen Sinn für die Zeremonie und die Repräsentation verdanke, die Fähigkeit zur Andacht vor der religiösen Manifestation und vor der Parade des neunten November auf dem Roten Platz im Kreml, vor jedem Augenblick der menschlichen Geschichte, dessen Schönheit seiner Größe entspricht, und vor jeder Tradition, die ja zumindest eine Vergangenheit beweist. 
Die Sinnlosigkeit der letzten Jahre des Kaisers war zugleich auch ein Stück der Kindheit des Erzählers: Die Sinnlosigkeit der Majestät hat dem Kind Sinn gegeben, und die kalte Sonne der Habsburger war immerhin eine Sonne. Die Trauer um den Kaiser ist zugleich auch eine Trauer um den Verlust der eigenen Kindheit. Alles das wird im Präteritum erzählt. Auch in den folgenden Textteilen, wo einige Szenen von der Abreise des Kaisers nach Ischl erzählt werden, findet sich kein Perfekt. Eine kleine Szene zum Schluss handelt von einer Frau, die ein Gnadengesuch in den Wagen des Kaisers wirft. Sie wird von Polizisten ergriffen und abgeführt. Der letzte Absatz lautet:

Fort war der Wagen. Das gleichmäßige Getrappel der Pferde ging unter im Geschrei der Menge. Die Sonne war heiß und drückend geworden. Ein schwerer Sommertag brach an. Vom Turm schlug es acht. Der Himmel wurde tiefblau. Die Straßenbahnen klingelten. Die Geräusche der Welt erwachten.

Auch hier keine Spur eines Perfekts. Die Ausfahrt des Kaisers hat keine Spur hinterlassen, nichts, das man sich merken, das man beachten müsste, nichts, das irgendwelche Folgen hätte. Die „Moral der Geschichte“- es gibt sie nicht. Es bricht ganz einfach ein neuer Tag an mit dem Klingeln der Straßenbahnen und den Geräuschen der Welt. Die Erzählung verdämmert in der gleißenden Sonne des Tages. Sie geht einfach einem Ende zu.

13. Eigenzeitliche Textabschnitte sind sinnstiftende Formen sprachlicher Gestaltungen. Erzählen heißt vom Vergangenen berichten, und das Vergangene ist oft eine Verkleidung des Fiktiven. Vergangenes und Fiktives ist vom Erzählenden entfernt, beides durch einen „Erinnerungsschnitt“ vom Erzählerzeit/sprechpunkt entfernt. Aber alles Erzählte hat einen Grund, eine Ursache, erzählt zu werden, und es steuert oft auf diesen Grund und diese Ursache im Lauf der Erzählung immer wieder zu. Das sind die Momente, bei denen die literarische Eigenzeitlichkeit ins Spiel kommt. Diese Momente sind wohl nicht nur für die Literatursprache wichtig, sondern auch für das sprachliche Ausdrucksvermögen an sich: Vielleicht kann man auch die sprachgeschichtliche Tatsache, dass sich Perfektformen im Lauf der Sprachgeschichte immer wieder erneuern, damit verbinden. Heimito von Doderer (Die Wiederkehr der Drachen, 22) hat diese Zusammenschau der Zeitebenen so beschrieben: 
Es ist das Gesetz von der „Symbiose der Zeiten“, wie es A.P. Gütersloh nennt: daß nämlich nichts, was war, durch nichts, was inzwischen geschehen ist, sich abhalten läßt, zu sein. Anders, und sozusagen massiv, formuliert: Jede einmal ausgespielte Karte bleibt auf irgendeine Weise im Spiel. So in der Erdgeschichte, so im geschichtlichen, so in unserem persönlichen Leben: auch hier staut sich das Volk des Gewesenen in dichtem und buntem Gedränge hinter den Kulissen der jetzt eben gespielten Szene und in den Gängen zwischen jenen, bereit, hervorzubrechen und die Bühne zu überschwemmen, alle Handlung an sich zu reißen.

\section{Quellen:}

Heimito von Doderer 1970 Die Wiederkehr der Drachen. Aufsätze/Traktate/ Reden, München: Biederstein.

Walter Jens 1963 „Bericht über Hattington“, in: Walter Jens, Herr Meister.

Dialog über einen Roman, München: Piper, 32-38.

Joseph Roth 1956 „Seine k. und k. apostolische Majestät“, in: Joseph Roth, Werke in drei Bänden, Köln-Berlin, Bd. 2, 328-333.

\section{Literatur:}

Comrie, Bernard 1985 Tense, Cambridge: Cambridge University Press.

Confais, Jean-Paul 1995 Temps-mode-aspect, Toulouse: Presses Universitaires du Mirail.

d'Alquen, Richard 1997 Time, mood, and aspect in German tense, Frankfurt/ Main - Wien: Lang.

Duden 1959 Grammatik der deutschen Gegenwartssprache, Mannheim: Bibliographisches Institut.

- 2005 Die Grammatik, Mannheim: Bibliographisches Institut Brockhaus.

Engel, Ulrich 1992 „Der Satz und seine Bausteine“, in: Vilmos Ágel / Regina Hessky (Hgg.), Offene Fragen - offene Antworten in der Sprachgermanistik, Tübingen: Niemeyer, 53-76.

- 2004 Deutsche Grammatik, München: iudicium.

Harweg, Roland 1994 Studien über Zeitstufen und ibre Aspektualität, Bochum: Brockmeyer. 
Klein, Wolfgang 1994 Time in language, London: Routledge.

Marschall, Matthias 1995 Textfunktionen der deutschen Tempora, Genf: Slatkine.

Nowotny, Helga 1993 Eigenzeit, Frankfurt/Main: Suhrkamp.

Quintin, Hervé/Margarete Najar/Stephanie Genz (Hgg.) 1997 Temporale Bedeutungen - Temporale Relationen, Tübingen: Stauffenburg.

Reichenbach, Hans 1966 [1947] Elements of Symbolic Logic, New York London: The Free Press.

Schecker, Michael/Elisenda Padros/Thomas Jechle 1997 „Textgliederung - und was sie leistet: Empirische Analysen zur Funktion der Vergangenheitstempora im alltäglichen Standarddeutsch“, in: Quintin/Najar/ Genz 1997, 167-177.

Thieroff, Rolf 1992 Das finite Verb im Deutschen. Tübingen: Narr.

Uexküll, Jakob von/Georg Kriszat 1956 Streifzüge durch die Umwelten von Tieren und Menschen, Hamburg: Rowohlt.

Vater, Heinz 1997 „Temporale und textuelle Funktionen deutscher Tempora“, in: Quintin/Najar/Genz 1997, 23-40.

Welke, Klaus 2005 Tempus im Deutschen, Berlin: de Gruyter. 
